\title{
Equilibrium Customer Strategies in the Single-Server Constant Retrial Queue with Breakdowns and Repairs
}

\author{
Zhengwu Zhang, Jinting Wang, and Feng Zhang \\ Department of Mathematics, Beijing Jiaotong University, Beijing 100044, China \\ Correspondence should be addressed to Jinting Wang; jtwang@bjtu.edu.cn
}

Received 23 September 2013; Accepted 28 November 2013; Published 2 January 2014

Academic Editor: Carsten Proppe

Copyright (C) 2014 Zhengwu Zhang et al. This is an open access article distributed under the Creative Commons Attribution License, which permits unrestricted use, distribution, and reproduction in any medium, provided the original work is properly cited.

\begin{abstract}
We consider a single-server constant retrial queueing system with a Poisson arrival process and exponential service and retrial times, in which the server may break down when it is working. The lifetime of the server is assumed to be exponentially distributed and once the server breaks down, it will be sent for repair immediately and the repair time is also exponentially distributed. There is no waiting space in front of the server and arriving customers decide whether to enter the retrial orbit or to balk depending on the available information they get upon arrival. In the paper, Nash equilibrium analysis for customers' joining strategies as well as the related social and profit maximization problems is investigated. We consider separately the partially observable case where an arriving customer knows the state of the server but does not observe the exact number of customers waiting for service and the fully observable case where customer gets informed not only about the state of the server but also about the exact number of customers in the orbit. Some numerical examples are presented to illustrate the effect of the information levels and several parameters on the customers' equilibrium and optimal strategies.
\end{abstract}

\section{Introduction}

Queueing systems in which arriving customers who find the server occupied may retry for service after a period of time are called retrial queues or queues with repeated orders. Among trials, a customer is said to be in "orbit". The retrial queueing system has been studied extensively due to its wide applications in telephone switching systems, telecommunication networks, and computer networks. The retrial queueing literature is quite extensive. For a recent account, the readers are referred to the books of Falin and Templeton [1] and Artalejo and Gómez-Corral [2] in which they have summarized the main models and methods thoroughly.

In the retrial queueing literature, the vast majority of articles assume that each customer seeks for service independently of other customers in orbit after a random time. In such cases, the total retrial rate of the system depends on the number of retrial customers in orbit. Nevertheless, in reality there are other types of queueing situations in which the repeated customers form a queue in orbit and only the head customer of the orbit can request a service after a random retrial time. This type of retrial discipline is called "constant retrial policy" and it arises from some applications in the computer and communication networks where the retrial rate is controlled by some automatic mechanism. It was introduced in Fayolle [3], who studied a telephone exchange model as an $\mathrm{M} / \mathrm{M} / 1$ retrial queue in which the orbiting customer who finds the server unavailable joins the tail of the queue. Farahmand [4] called this discipline a retrial queue with FCFS orbit. Later on, both retrial policies are incorporated by assuming the linear retrial policy introduced in Artalejo and Gómez-Corral [5].

Recently, Economou and Kanta [6] studied the equilibrium customer strategies and the social and profit maximization problems in the constant retrial system. They investigated two information cases: the unobservable case where the customers know only the state of the server (busy or idle) and the observable case where they also get informed about the number of customers in the retrial orbit. The customers' behavior is taken into account to identify the Nash 
equilibrium joining strategies. It differs from equilibrium analysis of retrial systems with the classical retrial policy; see, for instance, Kulkarni [7], Elcan [8], Hassin and Haviv [9], and Zhang et al. [10] and Wang et al. [11], among others. However, although we have found several retrial models with servers subject to breakdowns that consider orbits as FCFS queues, for example, in Atencia et al. [12], and Li and Zhao [10]. To the authors' knowledge, no work has been done on such queueing systems from an economic view. Thus, in this paper, we propose to study such an $M / M / 1$ retrial queue with the constant retrial policy and server's breakdowns and repairs as an extension of the paper considered by Economou and Kanta [6] who have also considered the observable single-server queue with breakdowns and repairs, see [13]. To this end, the methodology will be based on the gametheoretic analysis and optimization.

This paper is organized as follows. In Section 2, we give the model description and the natural reward-cost structure. In Sections 3 and 4, we determine Nash equilibrium, social, and profit maximization strategies for joining the retrial orbit in the partially observable case and the fully observable case, respectively. In Section 5, some numerical examples are given to illustrate the effect of the information levels and several parameters on the customers' strategies. Finally, in Section 6, some conclusions are given.

\section{Model Description}

We consider a single-server retrial queue without waiting line in which customers arrive according to a Poisson process at rate $\lambda$. The service times are assumed to be exponentially distributed with parameter $\mu$. During the busy periods, the server may break down and once breakdown occurs, the server will be repaired immediately. The customer being served will stay at the service area waiting for the server restored. The lifetime of the server is assumed to be exponentially distributed with parameter $\xi$, and the repair time is also exponentially distributed with parameter $\eta$.

Customers that find the server idle upon arrival will occupy the server immediately and start being served. On completion of the service, they leave the system. Upon arrival, if the server is busy, the arriving customers will join the retrial orbit and then retry for service according to an FCFS discipline. That is, only the customer at the head of the orbiting queue can repeat his request for service. We assume that retrial times are exponentially distributed with parameter $\alpha$. Obviously, the retrials can success only when the server is idle. In addition, newly arriving customers that find the server broken will leave the system and never enter the retrial orbit. Finally, it is assumed that the interarrival times, service times, lifetime of the server, repair times, and retrial times are mutually independent.

We denote the state of the system at time $t$ by a random vector $(I(t), N(t))$, where $I(t)$ denotes the state of the server, $0,1,2$ corresponding to the state of idle, busy or broken, respectively. And $N(t)$ is the number of customers in the orbit. Then $(I(t), N(t))$ is a continuous-time Markov chain

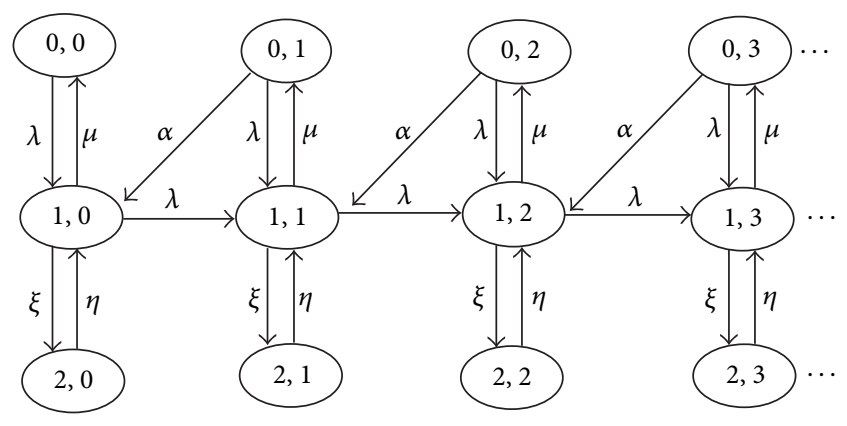

FIgURE 1: Transition rate diagram of the original model.

with state space $S=\{0,1,2\} \times\{0,1,2, \ldots\}$ and the related transition rates are given by

$$
\begin{array}{cl}
q_{(0, j)(1, j)}=\lambda, & j \geq 0, \\
q_{(1, j)(0, j)}=\mu, & j \geq 0, \\
q_{(1, j)(1, j+1)}=\lambda, & j \geq 0, \\
q_{(0, j)(1, j-1)}=\alpha, & j \geq 1, \\
q_{(1, j)(2, j)}=\xi, & j \geq 0, \\
q_{(2, j)(1, j)}=\eta, & j \geq 0 .
\end{array}
$$

The corresponding transition rate diagram is shown in Figure 1.

We focus on studying the behavior of customers when they are allowed to decide whether to join or balk based on the available information upon their arrival, including the queue length and/or the state of the server. In this paper, we assume that on completion of service, every customer receives a reward of $R$ units. Besides, we assume that there exists a waiting cost of $C$ units per time unit that is continuously accumulated from the time that the customer arrives at the system till the time he leaves the system after being served. We further assume that

$$
R>\frac{C(\xi+\eta)}{\mu \eta} .
$$

If this condition fails to hold, even the customer that finds the server idle will never enter the system, because of the negative net benefit. So, the above condition ensures that this queue is not empty.

We consider separately two information cases, that is, (1) partially observable case: arriving customers just observe the state of the server; (2) fully observable case: arriving customers get informed not only about the state of the server but also about the exact number of customers in the orbit. When the server is idle, customers will immediately enter the system, but when the server is busy, customers have to decide whether to enter the orbit or leave the system. We further assume that decisions are irrevocable: retrials of balking and reneging of entering customer are not allowed. 


\section{The Partially Observable Case}

As we have mentioned, a customer who finds the server idle will enter the system, because condition (7) ensures that his reward exceeds his expected waiting cost. In addition, the decision of the customer who finds the server idle will never be affected by the other customers. So, we just need to study the behavior of the customers that find the server busy upon arrival. We further assume that when an arriving customer finds the server busy, he will enter the orbit with probability $r$, because the customer does not know the exact number of customers in the orbit. In order to identify the individual equilibrium, social, and profit maximizing strategies, we need to examine the stationary probabilities of the system and then evaluate other key performances of the system. Based on the above assumptions, the transition rate of the corresponding Markov chain given in (3) should be substituted by

$$
q_{(1, j)(1, j+1)}=\lambda r, \quad j \geq 0 .
$$

Then, we have the following propositions.

Proposition 1. Consider the partially observable constant retrial queue with breakdowns and repairs, in which customers enter the system with probability $r$ whenever the server is busy, with probability 1 whenever the server is idle, and never enter the system whenever the server is broken. The stationary probabilities of the system are given by

$$
\begin{aligned}
& p_{0}(1)=\frac{\eta(\mu-\lambda r)}{\lambda(\xi+\eta)+\eta(\mu-\lambda r)}, \\
& p_{1}(1)=\frac{\lambda \eta}{\lambda(\xi+\eta)+\eta(\mu-\lambda r)}, \\
& p_{2}(1)=\frac{\lambda \xi}{\lambda(\xi+\eta)+\eta(\mu-\lambda r)} .
\end{aligned}
$$

Proof. Let $p_{i, n}(i=0,1,2 ; n=0,1,2, \ldots)$ denote the stationary probabilities of the system at state $(i, n)$, in which $i$ denotes the state of the server and $n$ denotes the number of customers in the orbit. According to the transition principle of the stationary probabilities, we get the following balance equations:

$$
\begin{gathered}
\lambda p_{0,0}=\mu p_{1,0} \\
(\lambda+\alpha) p_{0, n}=\mu p_{1, n}, \quad n \geq 1 \\
(\mu+\xi+\lambda r) p_{1,0}=\alpha p_{0,1}+\eta p_{2,0}+\lambda p_{0,0} \\
(\mu+\xi+\lambda r) p_{1, n}=\alpha p_{0, n+1}+\eta p_{2, n}+\lambda p_{0, n}+\lambda r p_{1, n-1} \\
\eta p_{2, n}=\xi p_{1, n}, \quad n \geq 0
\end{gathered}
$$

The transition rate diagram is shown in Figure 2.

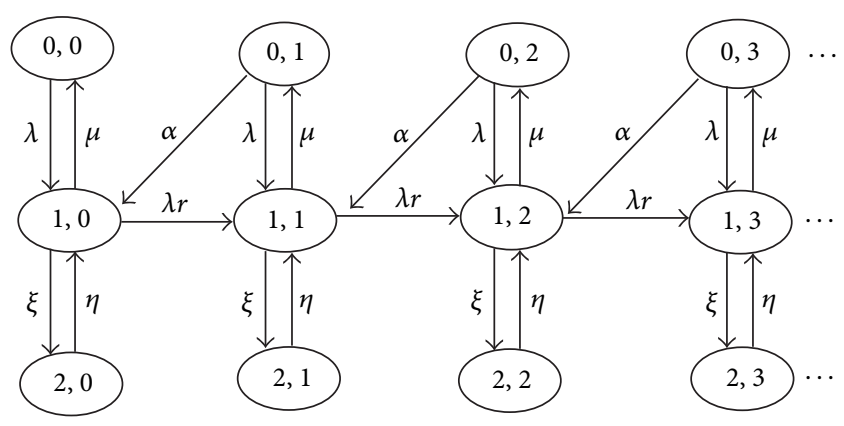

FIgURE 2: Transition rate diagram of the partially observable case.

In order to solve the stationary probabilities of the system, we define the following generating functions:

$$
p_{i}(z)=\sum_{n=0}^{\infty} p_{i, n} z^{n}
$$

where $i=0,1,2$. Multiplying (10) by $z^{0}$ and (11) by $z^{n}, n \geq 1$ and summing over $n$, we get

$$
(\lambda+\alpha) p_{0}(z)=\mu p_{1}(z)+\alpha p_{0,0} .
$$

Similarly, we can obtain $p_{1}(z)$ and $p_{2}(z)$ from (12)-(14)

$$
\begin{gathered}
{\left[-\lambda r z^{2}+z(\mu+\xi+\lambda r)\right] p_{1}(z)} \\
=(\lambda z+\alpha) p_{0}(z)+\eta z p_{2}(z)-\alpha p_{0,0}, \\
\eta p_{2}(z)=\xi p_{1}(z) .
\end{gathered}
$$

Using the above three equations, we yield

$$
\begin{aligned}
& p_{0}(z)=\frac{\alpha(\mu-\lambda r z)}{[\alpha \mu-(\lambda+\alpha) \lambda r z]} p_{0,0}, \\
& p_{1}(z)=\frac{\lambda \alpha}{[\alpha \mu-(\lambda+\alpha) \lambda r z]} p_{0,0}, \\
& p_{2}(z)=\frac{\xi \lambda \alpha}{\eta[\alpha \mu-(\lambda+\alpha) \lambda r z]} p_{0,0} .
\end{aligned}
$$

Using normalizing equation $p_{0}(1)+p_{1}(1)+p_{2}(1)=1$, we obtain

$$
p_{0,0}=\frac{\eta}{\alpha} \frac{\alpha \mu-(\lambda+\alpha) \lambda r}{\lambda(\xi+\eta)+\eta(\mu-\lambda r)} .
$$

Therefore, it is readily seen that the conclusion (9) can be derived based on the above results.

Remark 2. We need to determine the stable condition of this model in fact, from (10), (12), and (14), we obtain $\lambda r p_{1,0}=$ $\alpha p_{0,1}$. In addition, from (11), (13), and (14), we get $\lambda r p_{1, j}-$ $\alpha p_{0, j+1}=\lambda r p_{1, j-1}-\alpha p_{0, j}(j \geq 1)$. Proceeding to this recursive process, we get $\lambda r p_{1, j}=\alpha p_{0, j+1},(j \geq 1)$. Using (11) again, we obtain

$$
p_{0, j+1}=\frac{\lambda r(\lambda+\alpha)}{\alpha \mu} p_{0, j}, \quad j \geq 1 .
$$


So the system is stable if and only if

$$
\frac{\lambda r(\lambda+\alpha)}{\alpha \mu}<1
$$

Proposition 3. In the partially observable case, the expected overall queue length of this constant retrial queueing system and the expected mean sojourn time of an arriving customer that finds the server busy and decides to join the retrial orbit are given, respectively, by

$$
\begin{gathered}
E L_{\text {overall }}=\frac{\lambda^{2} r \mu \eta+\lambda \mu \alpha(\xi+\eta)}{[\lambda(\xi+\eta)+\eta(\mu-\lambda r)] \times[\alpha \mu-\lambda r(\lambda+\alpha)]} \\
E S_{\text {system }}=\frac{(\lambda+\alpha)(\xi+\eta)+\mu \eta}{\eta[\alpha \mu-(\lambda+\alpha) \lambda r]}+\frac{\eta+\xi}{\mu \eta}
\end{gathered}
$$

Proof. According to the PASTA property, we know that the total arrival rate in the orbit is given by

$$
\bar{\lambda}=\lambda r p_{1}(1) \text {. }
$$

Then the expected mean queue length in the orbit $\mathrm{EN}_{\text {orbit }}$ is given by

$$
\begin{aligned}
\mathrm{EN}_{\text {orbit }} & =\sum_{n=1}^{\infty} n\left(p_{0, n}+p_{1, n}+p_{2, n}\right) \\
& =p_{0}^{\prime}(1)+p_{1}^{\prime}(1)+p_{2}^{\prime}(1) .
\end{aligned}
$$

Differentiating (18) with respect to $z$ and taking $z=1$ yields

$$
\mathrm{EN}_{\text {orbit }}=\frac{\lambda^{2} r(\lambda+\alpha)(\xi+\eta)+\lambda^{2} r \mu \eta}{[\lambda(\xi+\eta)+\eta(\mu-\lambda r)] \times[\alpha \mu-\lambda r(\lambda+\alpha)]}
$$

Then, the expected sojourn time of an arriving customer that finds the server busy and decides to join in the retrial orbit is given by

$$
\mathrm{ES}_{\text {system }}=\frac{\mathrm{EN}_{\text {orbit }}}{\bar{\lambda}}+\frac{\xi+\eta}{\mu \eta}
$$

In the above equation, the first term $\mathrm{EN}_{\text {orbit }} / \bar{\lambda}$ equals to the mean waiting time in the orbit, and the second term $(\xi+$ $\eta) / \mu \eta$ means the generalized service time in a unreliable system, (see [14]). Using (24) and (26) yields (23). The expected overall queue length in the system $\mathrm{EL}_{\text {overall }}$ satisfies

$$
\begin{aligned}
& \mathrm{EL}_{\text {overall }} \\
& \qquad=p_{0}^{\prime}(1)+p_{1}^{\prime}(1)+p_{2}^{\prime}(1)+p_{1}(1)+p_{2}(1) \\
& \quad=\frac{\lambda^{2} r \mu \eta+\lambda \mu \alpha(\xi+\eta)}{[\lambda(\xi+\eta)+\eta(\mu-\lambda r)] \times[\alpha \mu-\lambda r(\lambda+\alpha)]} .
\end{aligned}
$$

In the following part of this paper, we assume that

$$
\frac{\lambda(\lambda+\alpha)}{\alpha \mu}<1
$$

This assumption originally comes from (21), but it has been modified slightly. This means the system is stable under any strategy $r$ followed by the customers. Under this condition, we are going to study the customers' equilibrium joining strategy. We have the following theorem.

Theorem 4. In the partially observable single-server constant retrial queue, we can derive a unique mixed equilibrium joining strategy "enter the orbit with probability $r_{e}$ when finding the server busy upon arrival," when condition (29) holds. The probability $r_{e}$ is given by

$$
r_{e}= \begin{cases}0, & \text { if } \frac{R}{C} \leq t_{L e} \\ r_{e}^{*}, & \text { if } t_{L e}<\frac{R}{C}<t_{U e} \\ 1, & \text { if } \frac{R}{C} \geq t_{U e}\end{cases}
$$

where

$$
\begin{gathered}
r_{e}^{*}=\frac{\alpha \mu}{\lambda(\lambda+\alpha)}-\frac{(\lambda+\alpha)(\xi+\eta)+\mu \eta}{\lambda \eta(\lambda+\alpha)}\left(\frac{R}{C}-\frac{\xi+\eta}{\mu \eta}\right)^{-1}, \\
t_{L e}=\frac{(\lambda+\alpha)(\xi+\eta)+\mu \eta}{\alpha \mu \eta}+\frac{\xi+\eta}{\mu \eta} \\
t_{U e}=\frac{(\lambda+\alpha)(\xi+\eta)+\mu \eta}{\eta(\mu \alpha-\lambda(\lambda+\alpha))}+\frac{\xi+\eta}{\mu \eta} .
\end{gathered}
$$

Proof. Suppose that all customers follow the same joining strategy $r$ when the server is busy at their arrival instant. Then we can get the expected net benefit of a customer who decides to enter the system

$$
\begin{aligned}
S_{e}(r) & =R-\mathrm{CES}_{\text {system }} \\
& =R-C\left[\frac{(\lambda+\alpha)(\xi+\eta)+\mu \eta}{\eta(\alpha \mu-(\lambda+\alpha) \lambda r)}+\frac{\eta+\xi}{\mu \eta}\right] .
\end{aligned}
$$

We observe that $S_{e}(r)$ is strictly decreasing whenever $r \epsilon$ $[0,1]$ and it has a unique maximum

$$
S_{e}(0)=R-C\left[\frac{(\lambda+\alpha)(\xi+\eta)+\mu \eta}{\alpha \mu \eta}+\frac{\eta+\xi}{\mu \eta}\right]
$$

and a unique minimum

$$
S_{e}(1)=R-C\left[\frac{(\lambda+\alpha)(\xi+\eta)+\mu \eta}{\eta(\alpha \mu-\lambda(\lambda+\alpha))}+\frac{\eta+\xi}{\mu \eta}\right] .
$$

Therefore, we consider the following cases.

(i) When $R / C \in\left((\eta+\xi) / \mu \eta, t_{L e}\right], S_{e}(r)$ is nonpositive whenever $r \in[0,1]$, so the best response is balking, and the equilibrium joining probability is $r_{e}=0$. 
(ii) When $R / C \in\left(t_{L e}, t_{U e}\right)$, there exists a unique solution of the equation $S_{e}(r)=0$, which is given by (31).

(iii) Similarly, when $R / C \in\left[t_{U e}, \infty\right)$, the net benefit is always positive, so we obtain the rest branch of the theorem.

Because of the monotonicity of the function $S_{e}(r)$, the best response is 1 whenever the joining probability $r$ is smaller than $r_{e}$. If $r>r_{e}$, the best response is 0 for the net benefit is negative. If $r=r_{e}$, any strategy is a best response and customers are indifferent between entering the system and leaving. This shows that an individual's best response is a decreasing function of the strategy selected by the other customers; that is the higher the joining probability of other customers, the lower the net benefit. Therefore, we have an "avoid-the-crowd" (ATC) situation.

We will proceed to determine the solutions of the social and profit maximization problems; that is, we need to find the optimal joining probabilities $r_{\text {soc }}$ and $r_{\text {prof }}$ that maximize the social net benefit and the administrator's profit per unit time. We firstly consider the social maximization problem. We have the following theorem.

Theorem 5. In the partially observable single-sever constant retrial queue, there exists a unique mixed joining strategy "enter the retrial orbit with probability $r_{\text {soc }}$ when the sever is busy" that maximizes the social net benefit per time unit, in which condition (29) holds. The probability $r_{\text {soc }}$ is given by

$$
r_{s o c}= \begin{cases}0, & \text { if } \frac{R}{C} \leq t_{L s o c} \\ r_{s o c}^{*}, & \text { if } t_{L s o c}<\frac{R}{C}<t_{U s o c} \\ 1, & \text { if } \frac{R}{C} \geq t_{U s o c}\end{cases}
$$

where

$$
\begin{gathered}
r_{s o c}^{*}=\frac{B_{0} \mu \alpha-\lambda(\xi+\eta)-\mu \eta}{\lambda(\lambda+\alpha) B_{0}-\lambda \eta} \\
B_{0}=\sqrt{\frac{\eta^{2}(\lambda R+C)}{C \alpha(\lambda+\alpha)}}, \\
t_{L s o c}=\frac{(\lambda+\alpha)[\lambda(\xi+\eta)+\mu \eta]^{2}-\mu^{2} \eta^{2} \alpha}{\lambda \mu^{2} \eta^{2} \alpha} \\
t_{U s o c}=\frac{\alpha(\lambda+\alpha)[\lambda(\xi+\eta)+\eta(\mu-\lambda)]^{2}-\eta^{2}[\mu \alpha-\lambda(\lambda+\alpha)]^{2}}{\lambda \eta^{2}[\mu \alpha-\lambda(\lambda+\alpha)]^{2}} .
\end{gathered}
$$

Proof. For a given joining strategy $r$, the system behaves stationarily when condition (29) holds. Customers that find the server busy will join the orbit with probability $r$. When all customers follow the same strategy $r$, the social net benefit per time unit is given by

$$
S_{\text {soc }}(r)=\lambda^{*}(r) R-\mathrm{CEL}_{\text {overall }}
$$

where $\lambda^{*}(r)$ denotes the mean effective arrival rate of the system, and $\mathrm{EL}_{\text {overall }}$ denotes the expected mean queue length of the system (including the customer in the server) under the strategy $r$. The mean effective arrival rate is given by

$$
\lambda^{*}(r)=\lambda p_{0}(1)+\lambda r p_{1}(1)=\frac{\lambda \mu \eta}{\lambda(\xi+\eta)+\eta(\mu-\lambda r)} .
$$

Using (22) and (43), social net benefit is given by

$$
\begin{aligned}
S_{\text {soc }}(r)= & \frac{\lambda \mu \eta}{\lambda(\xi+\eta)+\eta(\mu-\lambda r)} R-C \lambda \\
& \times \frac{\lambda \mu \eta r+\mu \alpha(\xi+\eta)}{[\lambda(\xi+\eta)+\eta(\mu-\lambda r)][\mu \alpha-\lambda r(\lambda+\alpha)]} .
\end{aligned}
$$

It can be written as

$$
S_{\text {soc }}(r)=\frac{\mu \eta(\lambda R+C)}{\lambda(\xi+\eta)+\eta(\mu-\lambda r)}-\frac{C \mu \alpha}{\mu \alpha-\lambda r(\lambda+\alpha)} .
$$

Differentiating the above equation with respect to $r$, we get

$$
\frac{d}{d r} S_{\text {soc }}(r)=\frac{\lambda \mu \eta^{2}(\lambda R+C)}{[\lambda(\xi+\eta)+\eta(\mu-\lambda r)]^{2}}-\frac{C \mu \alpha \lambda(\lambda+\alpha)}{[\mu \alpha-\lambda r(\lambda+\alpha)]^{2}},
$$

$$
\begin{aligned}
& \frac{d}{d r} S_{\text {soc }}(r)=0 \\
& \Longleftrightarrow \frac{\lambda(\xi+\eta)+\eta(\mu-\lambda r)}{\mu \alpha-\lambda r(\lambda+\alpha)}=\sqrt{\frac{\eta^{2}(\lambda R+C)}{C \alpha(\lambda+\alpha)}}
\end{aligned}
$$

From (46), we obtain

$$
\begin{gathered}
\left.\frac{d}{d r} S_{\text {soc }}(r)\right|_{r=0}=\frac{\lambda \mu \eta^{2}(\lambda R+C)}{[\lambda(\xi+\eta)+\mu \eta]^{2}}-\frac{C \mu \alpha \lambda(\lambda+\alpha)}{(\mu \alpha)^{2}}, \\
\left.\frac{d}{d r} S_{\text {soc }}(r)\right|_{r=1}= \\
-\frac{\lambda \mu \eta^{2}(\lambda R+C)}{[\lambda(\xi+\eta)+\eta(\mu-\lambda)]^{2}} \\
-\frac{C \mu \alpha \lambda(\lambda+\alpha)}{[\mu \alpha-\lambda(\lambda+\alpha)]^{2}} .
\end{gathered}
$$

Letting $\left.(d / d r) S_{\text {soc }}(r)\right|_{r=0}=0$ and $\left.(d / d r) S_{\text {soc }}(r)\right|_{r=1}=0$, we obtain $R / C=t_{L s o c}$ and $R / C=t_{U s o c}$, respectively. Then

$$
\begin{aligned}
& \left.\frac{d}{d r} S_{\text {soc }}(r)\right|_{r=0} \leq 0 \Longleftrightarrow \frac{R}{C} \leq t_{L s o c}, \\
& \left.\frac{d}{d r} S_{\text {soc }}(r)\right|_{r=1} \geq 0 \Longleftrightarrow \frac{R}{C} \geq t_{U s o c} .
\end{aligned}
$$

Therefore, we consider the following situations.

(i) When $R / C \leq t_{L s o c}$, function $S_{\text {soc }}(r)$ is decreasing whenever $r \in[0,1]$, so the best response is balking; then $r_{\text {soc }}=0$. 
(ii) When $t_{L s o c}<R / C<t_{U s o c}$, there exists a positive number $r_{0}$, so that function $S_{\text {soc }}(r)$ increases when $r \in\left(0, r_{0}\right]$ and decreases when $r \in\left[r_{0}, 1\right)$ then $r_{0}$ is the point that maximizes the function $S_{\text {soc }}(r)$. By solving (47), we know that the unique maximum of $S_{\text {soc }}(r)$ is attained at $\left(B_{0} \mu \alpha-\lambda(\xi+\eta)-\mu \eta\right) /\left(\lambda(\lambda+\alpha) B_{0}-\lambda \eta\right)$. Therefore, $r_{\text {soc }}$ given by (38) is the social maximizing strategy.

(iii) When $R / C \geq t_{U \text { soc }}$, function $S_{\text {soc }}(r)$ is increasing whenever $r \in[0,1]$, so the best response is 1 .

We now compare the optimal joining strategy of the social maximization problem and the individual equilibrium joining strategy. Their relation is given by the following theorem.

Theorem 6. In our single-server constant retrial rate queue with breakdowns and repairs, the joining probabilities $r_{e}$ and $r_{\text {soc }}$ are ordered as

$$
r_{s o c} \leq r_{e}
$$

Proof. When the stable condition (29) holds, it is easy to show that $t_{L e}<t_{L s o c}<t_{U e}<t_{U s o c}$, where $t_{L e}, t_{L s o c}, t_{U e}, t_{U s o c}$ are defined in Theorems 4 and 5 . We consider the following cases.

(i) If $R / C \leq t_{L e}$, according to (30) and (37), $r_{\text {soc }}=r_{e}=0$.

(ii) If $t_{L e}<R / C \leq t_{L s o c}$ then, $r_{e} \in(0,1)$ and $r_{\text {soc }}=0$. So, $r_{\mathrm{soc}}<r_{e}$.

(iii) If $t_{L \text { soc }}<R / C<t_{U e}$, then $r_{e} \in(0,1)$ and $r_{\text {soc }} \in(0,1)$. But we can get that $r_{\mathrm{soc}}<r_{e}$ after some algebra.

(iv) If $t_{U e} \leq R / C<t_{U \text { soc }}$ then, $r_{e}=1$ and $r_{\text {soc }} \in(0,1)$. Therefore, $r_{\text {soc }}<r_{e}$ is obviously satisfied.

(v) Finally, if $R / C \geq t_{U s o c}$, then $r_{e}=r_{\text {soc }}=1$.

From the above analysis, we complete the proof.

The above result can be explained by the perspective of an economic viewpoint. In the process of individual equilibrium analysis, we notice that customers will enter the orbit as long as their net benefit is nonnegative. Then, individual's rational behavior causes excessive use of the resource in equilibrium; that is to say, individual's optimization leads to the system having more congestion than what is socially desirable, which is the appearance of the negative externality.

Having considered the individual equilibrium strategy and social maximization problem, we will further identify the profit optimization joining strategy. In our model, we suppose that every customer will be imposed an entrance fee $p$ by the administrator. By imposing the entrance fee, customers' reward decreases to $R-p$; then their strategy will differ. We will determine the strategy $r_{\text {prof }}$ that maximizes the administrator's profit per time unit. The result is given by the following.
Theorem 7. In the partially observable single-server constant retrial queue, we can derive a unique mixed joining strategy "enter the orbit with probability $r_{\text {prof }}$ when the server is busy" that maximizes the administrator's net profit per time unit when condition (29) holds. The probability $r_{\text {prof }}$ is given by

$$
r_{\text {prof }}= \begin{cases}0, & \text { if } \frac{R}{C} \leq t_{\text {Lprof }} ; \\ r_{\text {prof }}^{*}, & \text { if } t_{\text {Lprof }}<\frac{R}{C}<t_{\text {Uprof }} ; \\ 1, & \text { if } \frac{R}{C} \geq t_{\text {Uprof }},\end{cases}
$$

where

$$
\begin{gathered}
r_{p r o f}^{*}=\frac{C_{0} \mu \alpha-\lambda(\xi+\eta)-\mu \eta}{\lambda(\lambda+\alpha) C_{0}-\lambda \eta} \\
C_{0}=\sqrt{\frac{\eta^{2}\left(\lambda R^{\prime}+C\right)}{C(\lambda+\alpha)^{2}}} \\
R^{\prime}=R-C \frac{\xi+\eta}{\mu \eta} \\
t_{\text {Lprof }} \\
=\frac{(\lambda+\alpha)^{2}[\lambda(\xi+\eta)+\mu \eta]^{2}+\lambda \mu \eta \alpha^{2}(\xi+\eta)-\mu^{2} \eta^{2} \alpha^{2}}{\lambda \mu^{2} \eta^{2} \alpha^{2}} \\
t_{\text {Uprof }}=\left(\mu(\lambda+\alpha)^{2}[\lambda(\xi+\eta)+\eta(\mu-\lambda)]^{2}\right. \\
\left.+\left[\lambda \eta(\xi+\eta)-\mu \eta^{2}\right][\mu \alpha-\lambda(\lambda+\alpha)]^{2}\right) \\
\times\left(\lambda \mu \eta^{2}[\mu \alpha-\lambda(\lambda+\alpha)]^{2}\right)^{-1}
\end{gathered}
$$

Proof. For a given joining strategy $r$, we define the function $S_{\text {prof }}(r)$ to be the administrator's net profit per time unit. Let $\lambda^{*}(r)$ be the mean effective arrival rate in the system; we further assume that the entrance fee that the administrator imposes on customers is $p(r)$. Then, we have

$$
S_{\text {prof }}(r)=\lambda^{*}(r) p(r)
$$

where $\lambda^{*}(r)$ is given by (43). In order to obtain $S_{\text {prof }}(r)$, we need to determine the entrance fee $p(r)$. Noticing (34), we yield

$$
R-p(r)=C\left(\frac{(\lambda+\alpha)(\xi+\eta)+\mu \eta}{\eta[\alpha \mu-\lambda r(\lambda+\alpha)]}+\frac{\xi+\eta}{\mu \eta}\right)
$$

from which we obtain

$$
p(r)=R-C \frac{\xi+\eta}{\mu \eta}-C \frac{(\lambda+\alpha)(\xi+\eta)+\mu \eta}{\eta[\alpha \mu-\lambda r(\lambda+\alpha)]} .
$$

Because if the entrance fee $p<p(r)$, the administrator can improve their net benefit through increasing the entrance fee, but if $p>p(r)$, the individual's net benefit is negative, 
customers will balk. So, (59) always holds whenever $r \in[0,1]$. Therefore, the net profit of the administrator can be calculated as follows:

$$
\begin{aligned}
S_{\text {prof }}(r) & =\lambda^{*}(r) p(r) \\
& =\frac{\mu \eta\left(\lambda R^{\prime}+C\right)}{\lambda(\xi+\eta)+\eta(\mu-\lambda r)}-C \frac{\mu(\lambda+\alpha)}{\mu \alpha-\lambda r(\lambda+\alpha)},
\end{aligned}
$$

where $R^{\prime}$ is given by (55). Differentiating the equation with respect to $r$, we get

$$
\begin{aligned}
\frac{d}{d r} S_{\text {prof }}(r)= & \frac{\lambda \mu \eta^{2}\left(\lambda R^{\prime}+C\right)}{[\lambda(\xi+\eta)+\eta(\mu-\lambda r)]^{2}} \\
& -\frac{C \mu \lambda(\lambda+\alpha)^{2}}{[\mu \alpha-\lambda r(\lambda+\alpha)]^{2}}, \\
\frac{d}{d r} S_{\text {prof }}(r)=0 & \\
& \Longleftrightarrow \frac{\lambda(\xi+\eta)+\eta(\mu-\lambda r)}{\mu \alpha-\lambda r(\lambda+\alpha)}=\sqrt{\frac{\eta^{2}\left(\lambda R^{\prime}+C\right)}{C(\lambda+\alpha)^{2}} .}
\end{aligned}
$$

The rest of the proof can proceed along the same line with the proof of Theorem 5 .

Now, we are going to compare the joining probabilities when considering the social and profit maximization problems. The result is given by the following theorem.

Theorem 8. In our model when condition (29) holds, the optimal joining probabilities $r_{s o c}$ and $r_{\text {prof }}$ are ordered as

$$
r_{\text {prof }} \leq r_{\text {soc }} \text {. }
$$

Proof. Firstly, we proceed to compare the relations among $t_{L s o c}, t_{U s o c}, t_{L p r o f}, t_{U \text { prof }}$. Rewrite the expressions of $t_{L \text { soc }}, t_{U \text { soc }}, t_{L \text { prof }}, t_{U \text { prof }}$ given in Theorems 5 and 7:

$$
\begin{gathered}
t_{L s o c}=\frac{(\lambda+\alpha)[\lambda(\xi+\eta)+\mu \eta]^{2}}{\lambda \mu^{2} \eta^{2} \alpha}-\frac{1}{\lambda}, \\
t_{U \text { soc }}=\frac{\alpha(\lambda+\alpha)[\lambda(\xi+\eta)+\eta(\mu-\lambda)]^{2}}{\lambda \eta^{2}[\mu \alpha-\lambda(\lambda+\alpha)]^{2}}-\frac{1}{\lambda}, \\
t_{L \text { prof }}=\frac{(\lambda+\alpha)^{2}[\lambda(\xi+\eta)+\mu \eta]^{2}}{\lambda \mu^{2} \eta^{2} \alpha^{2}}+\frac{\xi+\eta}{\mu \eta}-\frac{1}{\lambda}, \\
t_{U \text { prof }}=\frac{(\lambda+\alpha)^{2}[\lambda(\xi+\eta)+\eta(\mu-\lambda)]^{2}}{\lambda \eta^{2}[\mu \alpha-\lambda(\lambda+\alpha)]^{2}}+\frac{\xi+\eta}{\mu \eta}-\frac{1}{\lambda} .
\end{gathered}
$$

It is readily shown that $t_{L \text { soc }}<t_{L \text { prof }}$ and $t_{U \text { soc }}<t_{U \text { prof }}$. But the relation between $t_{U \text { soc }}$ and $t_{L \text { prof }}$ is undefined. Two cases may take place; that is, $t_{U \text { soc }}<t_{L \text { prof }}$ or $t_{U \text { soc }} \geq t_{L \text { prof }}$. We consider the situation when $t_{U \text { soc }} \geq t_{L \text { prof }}$, the other case can be analyzed in the same way. Then, we consider the following cases. (i) If $R / C \in\left[(\xi+\eta) / \mu \eta, t_{L s o c}\right)$ then, $r_{\text {soc }}=r_{\text {prof }}=0$.

(ii) If $R / C \in\left[t_{L s o c}, t_{L \text { prof }}\right)$, then $r_{\text {soc }} \in(0,1), r_{\text {prof }}=0$.

(iii) If $R / C \in\left[t_{L \text { prof }}, t_{U \text { soc }}\right)$, then $r_{\text {soc }} \in(0,1)$ and $r_{\text {prof }} \in$ $(0,1)$. We can take a close look at the expressions of $r_{\text {soc }}, r_{\text {prof }}$ that are presented in Theorems 5 and 7 . We define a function

$$
g(x)=\frac{x \mu \alpha-\lambda(\xi+\eta)-\mu \eta}{\lambda(\lambda+\alpha) x-\lambda \eta} .
$$

Differentiating the above function with respect to $x$, we obtain

$$
g^{\prime}(x)=\frac{\lambda^{2}((\lambda+\alpha)(\xi+\eta)+\mu \eta)}{(\lambda(\lambda+\alpha) x-\lambda \eta)^{2}} .
$$

It means that function $g(x)$ increases with respect to $x$. On the other hand, we can easily derive $B_{0}>C_{0}$, where $B_{0}$ and $C_{0}$ are given in Theorems 5 and 7. Then, we obtain $r_{\text {prof }}<r_{\text {soc }}$.

(i) If $R / C \in\left[t_{U \text { soc }}, t_{U \text { prof }}\right)$, then $r_{\text {soc }}=1$ and $r_{\text {prof }} \in(0,1)$.

(ii) If $R / C \in\left[t_{U \text { soc }}, \infty\right)$, then $r_{\text {soc }}=1$ and $r_{\text {prof }}=1$.

We obtain $r_{\text {soc }} \geq r_{\text {prof }}$ from the above analysis.

Remark 9. Economou and Kanta [6] have studied the individual equilibrium strategy and social and profit maximization problems in the single-server constant retrial queue in the partially observable case. In our model, if we let $\xi=0$, we can derive the same results as given in [6]. This is not accidentally happened, because when $\xi=0$, breakdowns will never occur; then these two models are equivalent. In addition, notice that customers' strategies $r_{e}, r_{\text {soc }}, r_{\text {prof }}$ are functions of $\xi / \eta$ where other parameters are fixed. It means that $r_{e}, r_{\text {soc }}, r_{\text {prof }}$ only relate to $\xi / \eta$, whatever was the value $\xi$ and $\eta$ we take in this model.

Remark 10. Another case is that $\lambda(\lambda+\alpha) / \alpha \mu \geq 1$ is left to be considered. In this situation, customers' joining strategies will be less than 1 when considering the equilibrium, social, and profit maximization problems, otherwise, the server will behave unstably. That is to say, we have the following joining strategies:

$$
\begin{gathered}
r_{e}= \begin{cases}0, & \text { if } \frac{R}{C} \leq t_{L e} ; \\
r_{e}^{*}, & \text { if } \frac{R}{C}>t_{L e},\end{cases} \\
r_{\text {soc }}= \begin{cases}0, & \text { if } \frac{R}{C} \leq t_{L s o c} ; \\
r_{\text {soc }}^{*}, & \text { if } \frac{R}{C}>t_{L s o c},\end{cases} \\
r_{\text {prof }}= \begin{cases}0, & \text { if } \frac{R}{C} \leq t_{L p r o f} ; \\
r_{\text {prof }}^{*}, & \text { if } \frac{R}{C}>t_{L p r o f},\end{cases}
\end{gathered}
$$

and the relation $r_{e} \geq r_{\text {soc }} \geq r_{\text {prof }}$ holds all the same. 
Till now, we have completed the analysis of the customers' strategies of the partially observable retrial queueing system. We will turn to the fully observable case.

\section{The Fully Observable Case}

We now focus on the fully observable case assuming customers observe not only the state of the server, but also the exact number of customers in the retrial orbit. Customer that finds the server idle will enter the system and begin to be served immediately, so this information is valuable only for the customer who finds the server busy. We know that after every service completion and there is no new customer arriving, the customer at the head of the orbit queue will successfully retry for service. That means customers in the orbit are served on an FCFS basis. Therefore, observing the position in the orbit, they can assess precisely whether to enter or balk.

Consider a tagged customer that finds the server busy upon arrival. This customer's mean overall sojourn time in the system is not affected by the joining/balking behavior of the other customers that find the server busy, because if the tagged customer joins the orbit queue at the $j$ th position, then the late customers who find the server busy will join the queue at the $j+1$ th, $j+2$ th,..., positions behind him in the orbit. So, his sojourn time does not depend on their decisions, but it depends on the arrival rate, because the newly arriving customer who finds the server idle will be served immediately.

To study the general threshold strategy adopted by all customers in the fully observable case, we will firstly consider the mean overall sojourn time of the customer who finds the server idle, busy or broken upon arrival. Let $T(1,0)$ be the mean sojourn time when the server is idle and there is no customers in the orbit at his arrival instant, and let $T(i, j)$ be the mean sojourn time of a tagged customer at the $j$ th position in the orbit, given that the server is at state $i$. When all customers follow the general strategy, the mean overall sojourn times $T(i, j)$ are given by

$$
\begin{gathered}
T(1,0)=\frac{\xi+\eta}{\mu \eta} \\
T(1, j)=\frac{1}{\mu+\xi}+\frac{\xi}{\mu+\xi} T(2, j)+\frac{\mu}{\mu+\xi} T(0, j), \quad j \geq 1 \\
T(0, j)=\frac{1}{\lambda+\alpha}+\frac{\lambda}{\lambda+\alpha} T(1, j)+\frac{\alpha}{\lambda+\alpha} T(1, j-1) \\
T(2, j)=\frac{1}{\eta}+T(1, j), \quad j \geq 0
\end{gathered}
$$

Let us consider a customer in the $j$ th position of the orbit when the server is busy. Then this customer has to wait for an exponentially distributed time with rate $\xi+\mu$ for the next event to occur, in which the server breaks down or the service is completed. With probability $\xi /(\xi+\mu)$ breakdown occurs; then the mean overall sojourn time becomes $T(2, j)$ and the tagged customer remains in the $j$ th position of the orbit. On the other hand, with probability $\mu /(\xi+\mu)$, the service is completed, then the mean overall sojourn time becomes $T(1, j-1)$ and the tagged customer moves to the $j-1$ th position. Through the above analysis, we obtain (69). Considering the other cases in the same line, we obtain the rest equations.

Solving the above equations, the mean overall sojourn times are given by

$$
\begin{gathered}
T(0, j)=j \frac{(\lambda+\alpha)(\xi+\eta)+\mu \eta}{\alpha \mu \eta}, \quad j \geq 1, \\
T(1, j)=j \frac{(\lambda+\alpha)(\xi+\eta)+\mu \eta}{\alpha \mu \eta}+\frac{\xi+\eta}{\mu \eta}, \quad j \geq 0, \\
T(2, j)=j \frac{(\lambda+\alpha)(\xi+\eta)+\mu \eta}{\alpha \mu \eta}+\frac{\mu+\xi+\eta}{\mu \eta}, \\
j \geq 0 .
\end{gathered}
$$

Then, we need to find the general equilibrium strategy followed by the customers when the server is busy. To ensure that customer will enter the orbit when finding the server busy and the queue in the orbit is empty, we further assume that $R-C T(1,1)>0$. The condition can be written as

$$
\frac{R}{C}>\frac{(\lambda+\alpha)(\xi+\eta)+\mu \eta}{\alpha \mu \eta}+\frac{\xi+\eta}{\mu \eta} .
$$

This means customers' reward should be larger in the fully observable case than in the former case even if the other systematic parameters are equal. We will identify the customers' equilibrium threshold strategy, and we have the following theorem.

Theorem 11. In the fully observable single-server constant retrial queue, there exists a unique threshold joining strategy "enter the retrial orbit if there are at most $n_{e}$ customers in the orbit, whenever finding the server busy," in which condition (73) holds. The threshold $n_{e}$ is given by

$$
n_{e}=\left\lfloor x_{e}\right\rfloor,
$$

where

$$
x_{e}=\frac{\alpha \mu \eta R-C \alpha(\xi+\eta)}{C[(\lambda+\alpha)(\xi+\eta)+\mu \eta]}-1
$$

This strategy is the unique equilibrium strategy among all possible strategies.

Proof. Consider a tagged customer that finds the system at the state $(1, j)$ upon arrival. If he decides to join, he goes directly to the orbit and occupies the $j+1$ th position. Then, his expected net benefit is $S_{e}(j)=R-C T(1, j+1)$, where

$$
S_{e}(j)=R-C\left[(j+1) \frac{(\lambda+\alpha)(\xi+\eta)+\mu \eta}{\alpha \mu \eta}+\frac{\xi+\eta}{\mu \eta}\right] \text {. }
$$




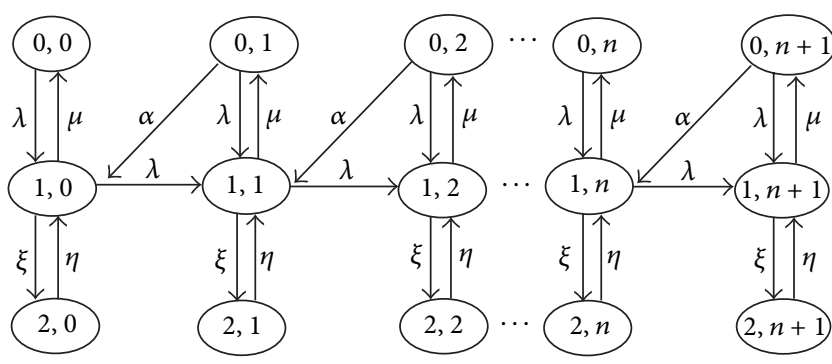

FiguRE 3: Transition rate diagram of the fully observable case.

The customer prefers to join if $S_{e}(j)>0$, and he is indifferent between joining and balking while $S_{e}(j)=0$ and prefers to balk if $S_{e}(j)<0$. A newly arriving customer will enter if and only if $j \leq n_{e}$ when finding the server busy. By solving inequality $S_{e}(j) \geq 0$, we obtain the result.

We will elucidate the uniqueness of the equilibrium threshold joining strategy followed by all customers. Indeed, if $x_{e}$ is an integer number, we just need to let $n_{e}=x_{e}$; then we know $S_{e}\left(n_{e}\right)=0$ and the mixed threshold strategy is that prescribes to enter if $n<n_{e}$, to balk if $n>n_{e}$ and be indifferent if $n=n_{e}$. When $x_{e}$ is any positive value, we let $n_{e}=\left\lfloor x_{e}\right\rfloor$. Hence, in this case, the equilibrium strategy is also unique.

We now turn our attention to the social and profit maximization problems and identify the thresholds $n_{\text {soc }}$ and $n_{\text {prof }}$ that maximize the social net benefit and the administrator's profit per time unit. To consummate this goal, we need to determine the stationary behavior of the system when all customers follow the same threshold strategy.

Suppose that all customers that find the server busy follow the same threshold strategies $n_{\text {soc }}$ and $n_{\text {prof }}$, respectively. That is to say, customers who find the sever busy will enter the system as long as the number of customers in the orbit is at most $n_{\text {soc }}$ when considering the social maximization problem, and there is at most $n_{\text {prof }}$ customers in the orbit when considering the administrator's optimization problem. Hence, the maximum number of customers in the orbit will never be larger than $n+1$ when customers follow the same threshold- $n$ strategy. Then we obtain a continuous-time Markov chain $(I(t), N(t))$ with state space $S=\{0,1,2\} \times$ $\{0,1,2, \ldots, n+1\}$, and its transition diagram is given by Figure 3.

The stationary distribution of the system, $\left(p_{i, j}(i, j) \in S\right)$, is given by the following proposition.

Proposition 12. Consider the fully observable single-server constant retrial queue with breakdowns and repairs, in which customers follow a threshold-n strategy; then the stationary distribution of the system is given by

$$
\begin{gathered}
p_{0,0}=B(n) \frac{\mu \alpha}{\lambda^{2}}, \\
p_{0, j}=B(n) \rho^{j-1}, \quad j=1,2, \ldots, n+1,
\end{gathered}
$$

$$
\begin{aligned}
& p_{1, j}=B(n) \frac{\alpha}{\lambda} \rho^{j}, \quad j=0,1,2, \ldots, n+1, \\
& p_{2, j}=B(n) \frac{\alpha}{\lambda} \frac{\xi}{\eta} \rho^{j}, \quad j=0,1,2, \ldots, n+1,
\end{aligned}
$$

where

$$
\rho=\frac{\lambda(\lambda+\alpha)}{\alpha \mu},
$$

$$
\begin{gathered}
B(n)=\left\{\frac{\mu \alpha}{\lambda^{2}}+\left(1+\rho+\cdots+\rho^{n}\right)+\frac{\alpha}{\lambda}\left(1+\rho+\cdots+\rho^{n+1}\right)\right. \\
\left.+\frac{\alpha \xi}{\lambda \eta}\left(1+\rho+\cdots+\rho^{n+1}\right)\right\}^{-1} .
\end{gathered}
$$

Proof. The stationary distribution is obtained from the following balance equations:

$$
\begin{gathered}
\lambda p_{0,0}=\mu p_{1,0}, \\
(\lambda+\alpha) p_{0, j}=\mu p_{1, j}, \quad j=1,2, \ldots, n+1, \\
\lambda p_{1, j}=\alpha p_{0, j+1}, \quad j=0,1,2, \ldots, n, \\
\eta p_{2, j}=\xi p_{1, j}, \quad j=0,1, \ldots, n+1, \\
(\mu+\xi) p_{1, n+1}=\eta p_{2, n+1}+\lambda p_{1, n}+\lambda p_{0, n+1} .
\end{gathered}
$$

Combining (80) with (81), we can obtain the recursive relation

$$
p_{1, j}=p_{1,0} \rho^{j}, \quad j=1,2, \ldots, n-1 .
$$

Then, we derive the expressions of $p_{2, j}$ from (82),

$$
p_{2, j}=\frac{\xi}{\eta} \rho^{j} p_{1,0}, \quad j=0,1, \ldots, n+1 .
$$

Using (80)-(82), we can get

$$
\begin{gathered}
p_{0,0}=p_{1,0} \frac{\mu \alpha}{\lambda^{2}}, \\
p_{0, j}=p_{1,0} \rho^{j-1}, \quad j=1,2, \ldots, n+1, \\
p_{1, j}=p_{1,0} \frac{\alpha}{\lambda} \rho^{j}, \quad j=0,1,2, \ldots, n+1, \\
p_{2, j}=p_{1,0} \frac{\alpha}{\lambda} \frac{\xi}{\eta} \rho^{j}, \quad j=0,1,2, \ldots, n+1 .
\end{gathered}
$$

Then, with the help of the normalizing equation $\sum_{j=0}^{n+1} p_{0, j}+$ $\sum_{j=0}^{n+1} p_{1, j}+\sum_{j=0}^{n+1} p_{2, j}=1$, we get the expression of $p_{1,0}$.

By considering the stationary distribution, we examine the social welfare maximization threshold strategy. We have the following result. 
Theorem 13. In our single-server constant retrial queue, in which the condition (73) holds, we define the following function:

$$
\begin{aligned}
f(x)= & \frac{(\mu \alpha+(\xi / \eta) \lambda \alpha)(x+1)}{\mu \alpha(1-\rho)} \\
& -\frac{\lambda(\lambda+\alpha \rho(1+(\xi / \eta)))\left(1-\rho^{x+1}\right)}{\mu \alpha(1-\rho)^{2}}-1 .
\end{aligned}
$$

If $f(0)>x_{e}$, then the customers' best response is balking. Otherwise, there exists a unique threshold- $n_{\text {soc }}$ strategy "enter the retrial orbit if the number of customers in the orbit is at most $n_{\text {soc }}$, whenever customers find the server busy" that maximizes the social net benefit per time unit. The threshold $n_{\text {soc }}$ is given by

$$
n_{s o c}=\left\lfloor x_{s o c}\right\rfloor,
$$

where $x_{\text {soc }}$ is the unique nonnegative root of the equation $f(x)=x_{e}$.

Proof. In order to determine the optimal threshold $n_{\text {soc }}$, we need to compute the effective arrival rate of the system and the expected mean sojourn time of the system. On the one hand, when all customers follow the same threshold- $n$ strategy, the effective arrival rate is given by

$$
\lambda(n)=\lambda\left(\sum_{j=0}^{n+1} p_{0, j}(n)+\sum_{j=0}^{n} p_{1, j}(n)\right) .
$$

On the other hand, by conditioning on the state found by a customer in the system upon arrival, we obtain the expected mean sojourn time if entering the system with threshold- $n$ policy

$$
E[S(n)]=A+B,
$$

where

$$
\begin{aligned}
A & =\sum_{j=0}^{n+1} \frac{p_{0, j}(n)}{\sum_{k=0}^{n+1} p_{0, k}(n)+\sum_{k=0}^{n} p_{1, k}(n)} \frac{\xi+\eta}{\mu \eta}, \\
B= & \sum_{j=0}^{n} \frac{p_{1, j}(n)}{\sum_{k=0}^{n+1} p_{0, k}(n)+\sum_{k=0}^{n} p_{1, k}(n)} \\
& \times\left(\frac{\xi+\eta}{\mu \eta}+(j+1) \frac{(\lambda+\alpha)(\xi+\eta)+\mu \eta}{\alpha \mu \eta}\right) .
\end{aligned}
$$

The expected social net benefit is given by

$$
S_{\text {soc }}(n)=\lambda(n) R-C \lambda(n) E[S(n)] .
$$

Using (90) and (91) and after some algebra, we obtain

$$
\begin{aligned}
S_{\mathrm{soc}}(n)= & C \frac{(\lambda+\alpha)(\xi+\eta)+\mu \eta}{\alpha \mu \eta} \\
& \times\left(\lambda(n)\left(x_{e}+1\right)-\lambda \sum_{j=0}^{n}(j+1) p_{1, j}(n)\right) .
\end{aligned}
$$

Next, we need to show that $S_{\text {soc }}(n)$ is unimodal and has only one maximal point. We firstly consider the increments $S_{\text {soc }}(n)-S_{\text {soc }}(n-1)$. It is not difficult to show that

$$
S_{\text {soc }}(n)-S_{\text {soc }}(n-1) \geq 0 \Longleftrightarrow f(n) \leq x_{e},
$$

where $f(n)$ is

$$
\begin{gathered}
f(n)=\frac{f_{1}(n)}{f_{2}(n)}-1 \\
f_{1}(n)=\lambda\left(\sum_{j=0}^{n}(j+1) p_{1, j}(n)-\sum_{j=0}^{n-1}(j+1) p_{1, j}(n-1)\right) \\
f_{2}(n)=\lambda(n)-\lambda(n-1) .
\end{gathered}
$$

Using the stationary distribution, we can simplify the expressions of (97) and (98) as follows:

$$
\begin{gathered}
f_{1}(n)=\frac{\alpha B(n) B(n-1) \rho^{n}}{\lambda^{2}(1-\rho)^{2}} \times D, \\
f_{2}(n)=\frac{\mu \alpha^{2} \rho^{n}}{\lambda^{2}} B(n) B(n-1),
\end{gathered}
$$

where

$$
\begin{aligned}
D=\{ & \left(\mu \alpha+\lambda \alpha \frac{\xi}{\eta}\right)(n+1)(1-\rho)-\lambda^{2} \\
& \left.\quad-\lambda \alpha \rho\left(1+\frac{\xi}{\eta}\right)+\lambda^{2} \rho^{n+1}+\lambda \alpha\left(1+\frac{\xi}{\eta}\right) \rho^{n+2}\right\} .
\end{aligned}
$$

Plugging (99) and (100) in (96) and replacing $n$ by $x$, we obtain (87). To prove the unimodality of $S_{\text {soc }}(n)$, we need to show that function $f(x)$ is increasing. We define the function $h:[0, \infty) \rightarrow R$ with

$$
h(x)=f(x)-x .
$$

We have

$$
\begin{aligned}
\frac{d^{2}}{d x^{2}} h(x)=\frac{[\lambda+\alpha \rho(1+(\xi / \eta))] \rho^{x+2}(\ln \rho)^{2}}{(\lambda+\alpha)(1-\rho)^{2}} & >0, \\
x & \geq 0 .
\end{aligned}
$$

Then, function $h(x)$ is a strictly convex function and there is no flex point with respect to $\rho$ and $x$. Therefore, $h(x)$ is increasing and so is $f(x)=h(x)+x$. Therefore, two cases may take place.

(i) $f(0)>x_{e}$; then $S_{\text {soc }}(n)$ is decreasing and customers who find the server busy will balk.

(ii) $f\left(n_{\text {soc }}\right) \leq x_{e}<f\left(n_{\text {soc }}+1\right)$; then we have $S_{\text {soc }}(n)-$ $S_{\text {soc }}(n-1) \geq 0$ for $n \leq n_{\text {soc }}$ and $S_{\text {soc }}(n)-S_{\text {soc }}(n-1)<0$ for $n>n_{\text {soc }}$. Then the maximum of $S_{\text {soc }}(n)$ occurs in $n_{\text {soc }}=\left\lfloor x_{\text {soc }}\right\rfloor$, in which $x_{\text {soc }}$ is the unique solution of the equation $f(x)=x_{e}$. 
The last purpose of this paper is to solve the profit maximization problem. By imposing an appropriate nonnegative admission fee $p$ on the customers, the administrator can maximize his net profit per time unit. We have the following theorem.

Theorem 14. In the fully observable single-server constant retrial queue with breakdowns and repairs, in which condition (73) holds, let

$$
\begin{aligned}
g(x)= & x+\mu\left(1-\rho^{x+1}\right) \\
& \times\left(1+(\lambda \xi / \mu \eta)-((\lambda+\alpha \rho(1+\xi / \eta)) /(\lambda+\alpha)) \rho^{x+2}\right) \\
& \times\left(\lambda \rho^{x}(1-\rho)^{2}\right)^{-1},
\end{aligned}
$$

if $g(0)>x_{e}$, then customers' best strategy is balking. Otherwise, there exists a unique threshold joining strategy "enter the retrial orbit when the number of customers in the orbit is at most $n_{\text {prof }}$, whenever finding the server busy" that maximizes the administrator's net profit per time unit. The threshold $n_{\text {prof }}$ is given by

$$
n_{\text {prof }}=\left\lfloor x_{\text {prof }}\right\rfloor
$$

where $x_{\text {prof }}$ is the unique nonnegative root of the equation $g(x)=x_{e}$.

Proof. We first need to determine the appropriate entrance fee $p$ that the administrator imposes on the customers in the system. As we have analyzed in Theorem 7 , the entrance fee $p$ also satisfies

$$
R-p(n)=C\left(\frac{\xi+\eta}{\mu \eta}+(n+1) \frac{(\lambda+\alpha)(\xi+\eta)+\mu \eta}{\alpha \mu \eta}\right) .
$$

From the above equation, we obtain

$$
p(n)=R-C\left(\frac{\xi+\eta}{\mu \eta}+(n+1) \frac{(\lambda+\alpha)(\xi+\eta)+\mu \eta}{\alpha \mu \eta}\right) .
$$

The administrator's profit per time unit is

$$
\begin{aligned}
S_{\text {prof }}(n)= & \lambda(n) p(n) \\
= & \lambda(n)\left(R-C\left(\frac{\xi+\eta}{\mu \eta}+(n+1)\right.\right. \\
& \left.\left.\times \frac{(\lambda+\alpha)(\xi+\eta)+\mu \eta}{\alpha \mu \eta}\right)\right) \\
= & C \frac{(\lambda+\alpha)(\xi+\eta)+\mu \eta}{\alpha \mu \eta} \lambda(n)\left(x_{e}-n\right) .
\end{aligned}
$$

We will proceed to prove the unimodality of the function $g(x)$. From (108), we get

$$
\frac{S_{\text {prof }}(n)}{S_{\text {prof }}(n-1)} \geq 1 \Longleftrightarrow g(n) \leq x_{e},
$$

where $g(n)=n+\lambda(n-1) /(\lambda(n)-\lambda(n-1))$. Simplifying (90) and using (98) and (100), we obtain the expression of $g(n)$; substituting $n$ by $x$, we obtain (100). We can examine the function $g(x)$ is increasing. Then we consider the following two cases.

(i) $g(0)>x_{e}$, then function $S_{\text {prof }}(n)$ is decreasing and the customers' best response is balking.

(ii) $g\left(n_{\text {prof }}\right) \leq x_{e}<g\left(n_{\text {prof }}+1\right)$; then $S_{\text {prof }}(n)-S_{\text {prof }}(n-$ $1) \geq 0$ for $n \leq n_{\text {prof }}$ and $S_{\text {prof }}(n)-S_{\text {prof }}(n-1)<0$ for $n>n_{\text {prof }}$. Then the maximum of $S_{\text {prof }}(n)$ is attained at $n_{\text {prof }}=\left\lfloor x_{\text {prof }}\right\rfloor$, where $x_{\text {prof }}$ is the unique solution of $g(x)=x_{e}$.

Remark 15. In this case, if we take a close look at the expressions of thresholds $n_{e}, n_{\text {soc }}$, and $n_{\text {prof }}$, we will find that these thresholds are also functions of $\xi / \eta$ when other parameters are fixed. Furthermore, we let $\xi$ equal to zero, then this case is equivalent to the model studied in [6], and we have the same results when counterpart problems are considered. So, it is also a special case of this model.

Now we finish the analysis of the single-server retrial queueing system with constant retrial rate in the partially and fully observable cases. We obtain the entrance probabilities in the partially observable case and the thresholds in the latter case. In the following section, we will present some numerical examples to support our results.

\section{Numerical Examples}

In this section, we will investigate the effects of the parameters on the customers' mixed strategies in this constant retrial queueing system. Figures 4, 5, and 6 present the influence of the parameters on the equilibrium joining probability $r_{e}$ and optimal entrance probabilities $r_{\text {soc }}$ and $r_{\text {prof }}$, where parameters $\lambda, \alpha$, and $\xi / \eta$ vary, respectively. In these figures, we can clearly see that $r_{e} \geq r_{\text {soc }} \geq r_{\text {prof }}$, which has been proved in Theorems 6 and 8.

Now, we pay our attention to the fully observable case. Figures 7-10 depict the equilibrium and optimal joining thresholds vary with respect to parameters $\lambda, \mu, \alpha$, and $\xi / \eta$. From these four figures, we can see $n_{\text {prof }} \leq n_{\text {soc }} \leq n_{e}$. Indeed, the relation $n_{\mathrm{soc}} \leq n_{e}$ is obvious from Theorems 11 and 13 . On the one hand, we can obtain that $h(0)=f(0)>0$, and because function $h(x)$ is increasing and $h\left(x_{\text {soc }}\right) \geq 0$, then we get $f\left(x_{\text {soc }}\right)-x_{\text {soc }} \geq 0$. But on the other hand, $f\left(x_{\text {soc }}\right)=x_{e}$ which means $x_{\text {soc }} \leq x_{e}$. Taking floors we obtain the result $n_{\mathrm{soc}} \leq n_{e}$. The relation $n_{\text {prof }} \leq n_{\mathrm{soc}}$ is not proved theoretically, but it can be traced numerically.

Figure 7 shows the thresholds when we consider the individual equilibrium, social, and profit maximization threshold 


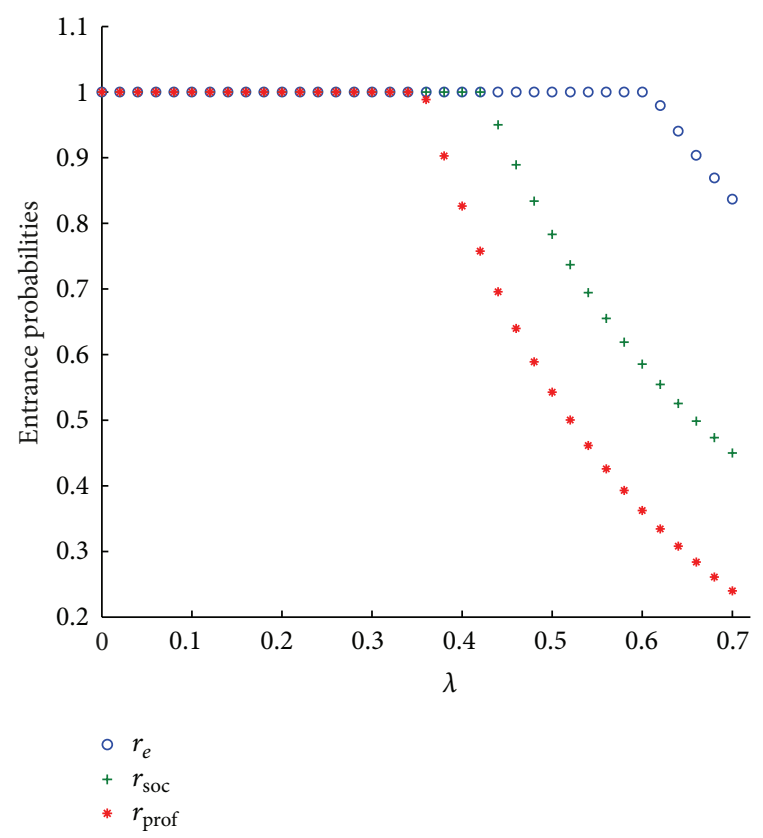

FIGURE 4: Individual equilibrium strategy and optimal entrance probabilities vary with respect to $\lambda$, for $\mu=1, \eta=1, \xi=0.02$, $\alpha=2, R=10$, and $C=1$.

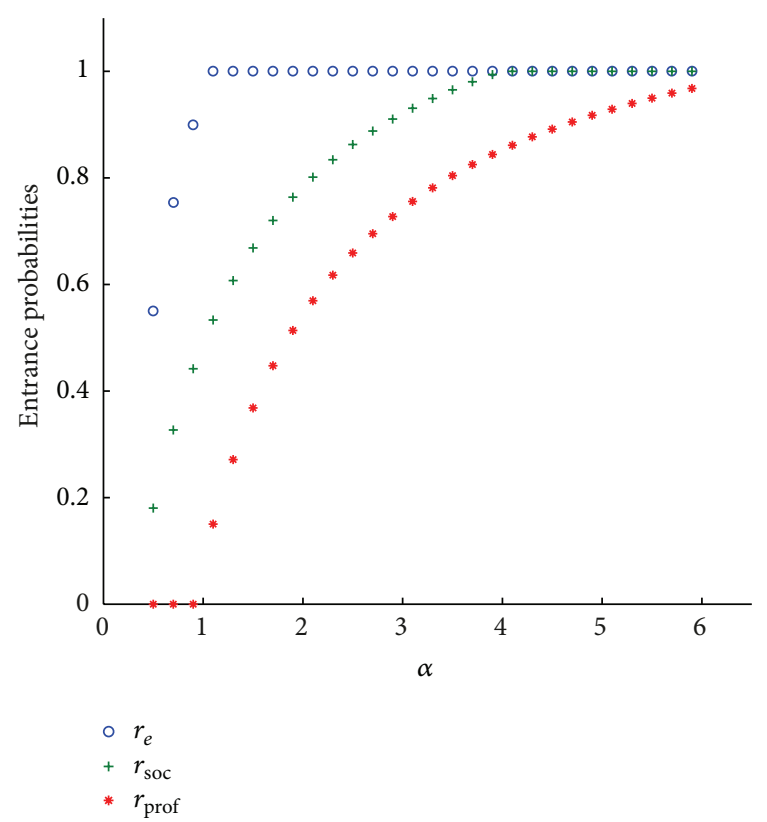

FIGURE 5: Individual equilibrium strategy and optimal entrance probabilities vary with respect to $\alpha, \lambda=0.5$, for $\mu=1, \xi=0.02$, $R=10$, and $C=1$.

strategies with respect to $\lambda$. We can see that the thresholds finally converge to 0 . On the one hand, it is true that when $\lambda$ becomes larger, there are more customers arriving per time unit. So customers in the orbit will wait longer to successfully retry for service. On the other hand, from (74) we can see that $n_{e}$ is decreasing with respect to $\lambda$. And because of the function $S_{\text {soc }}(n)$ and $S_{\text {prof }}(n)$, we can see that thresholds $n_{\text {soc }}$ and $n_{\text {prof }}$

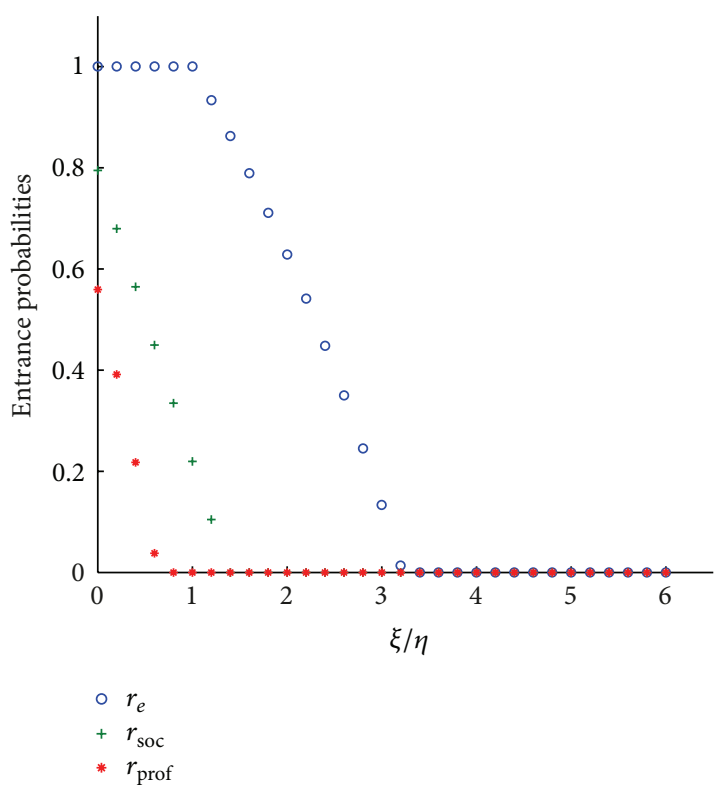

FIGURE 6: Individual equilibrium strategy and optimal entrance probabilities vary with respect to $\xi / \eta$, for $\mu=1, \lambda=0.5, \alpha=2$, $R=10$, and $C=1$.

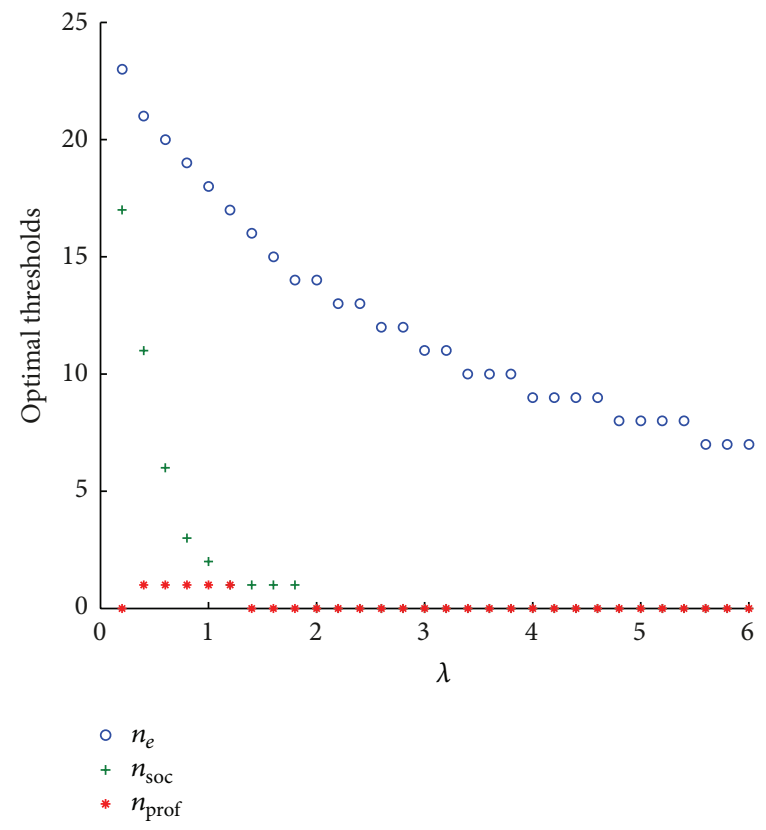

FIGURE 7: Individual equilibrium threshold and optimal thresholds vary with respect to $\lambda$, for $\xi=0.02, \mu=1, \eta=1, \alpha=2, R=40$, and $C=1$.

finally become zero for large value of $\lambda$. We have $n_{\text {prof }} \leq n_{\text {soc }}$ as $\lambda$ varies. When considering the variation of parameters $\mu$ and $\alpha$ in Figures 8 and 9 , taking mean service and retrial times of the system into consideration, we can easily explain the outcomes, and we also have $n_{\text {prof }} \leq n_{\text {soc }}$.

Figure 10 presents the thresholds vary with respect to $\xi / \eta$. On the one hand, from the expression of $n_{e}$ and functions 


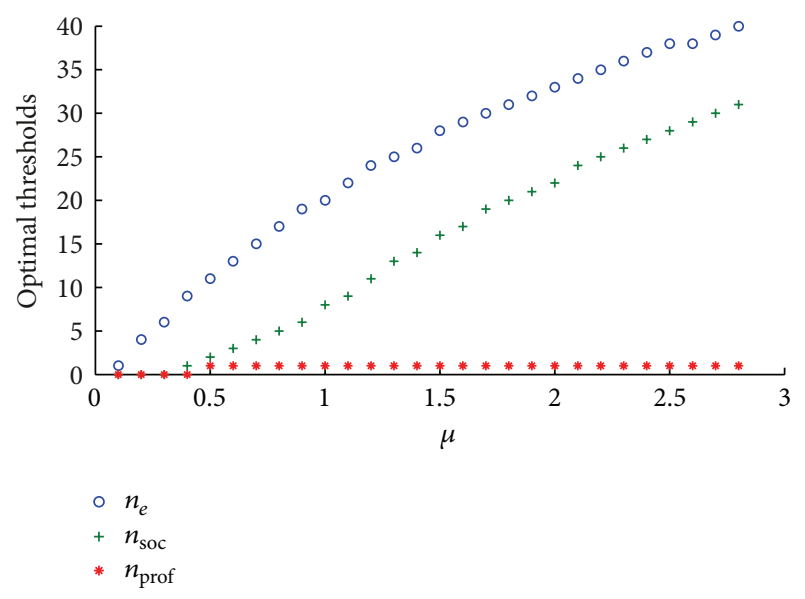

FIGURE 8: Individual equilibrium threshold and optimal thresholds vary with respect to $\mu$, for $\xi=0.02, \alpha=2, \eta=1, \lambda=0.5, R=40$, and $C=1$.

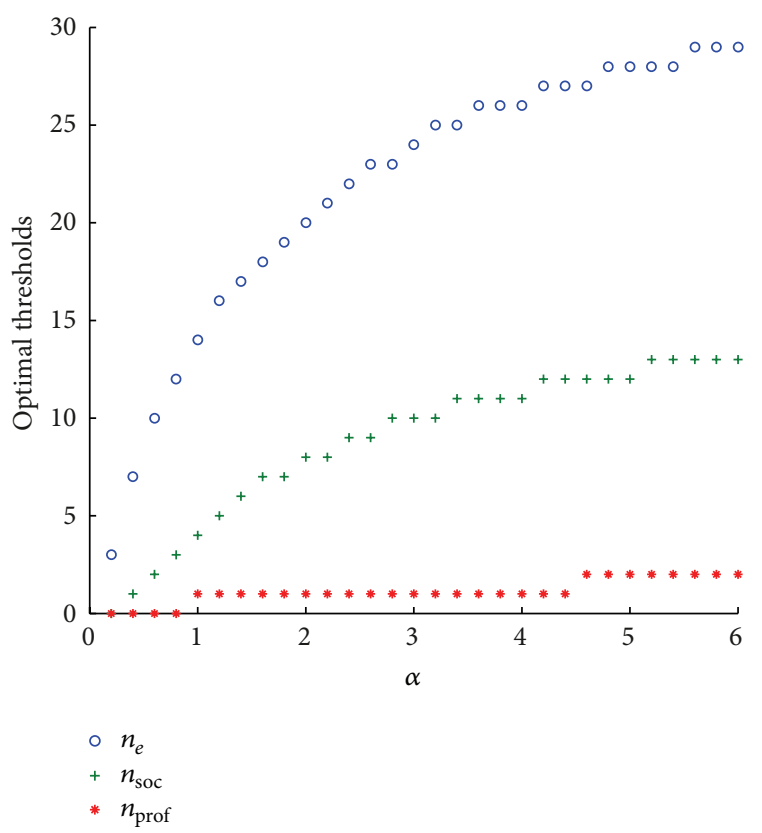

FIGURE 9: Individual equilibrium threshold and optimal thresholds vary with respect to $\alpha$, for $\xi=0.02, \mu=1, \eta=1, \lambda=0.5, R=40$, and $C=1$.

$S_{\text {soc }}(n)$ and $S_{\text {prof }}(n)$, we know that thresholds decrease to 0 when $\xi / \eta$ exceeds a positive number. On the other hand, when $\xi / \eta$ increases, it means that the lifetime of the system becomes shorter; then the customers in the orbit need to wait longer for the server restored. So, the thresholds decrease with respect to $\xi / \eta$. When $\xi / \eta$ varies, we can clearly see that $n_{\text {prof }} \leq n_{\text {soc }}$.

\section{Conclusions}

We have analyzed the equilibrium and optimal entrance probabilities in the partially observable case and the counterpart thresholds in the fully observable case. We have an

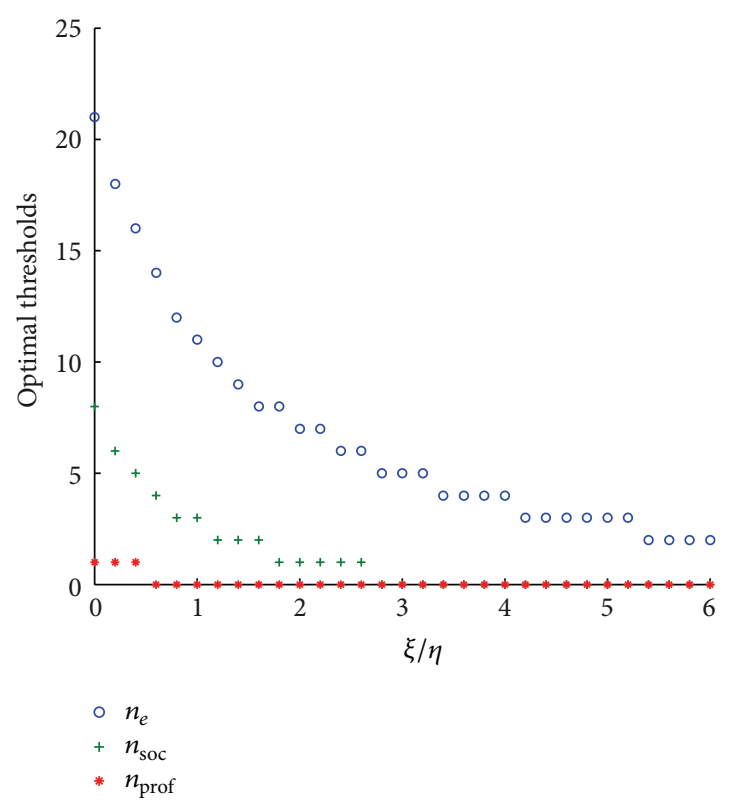

FIgURE 10: Individual equilibrium threshold and optimal thresholds vary with respect to $\xi / \eta$, for $\lambda=1, \mu=1, \alpha=2, R=40$, and $C=1$.

"avoid-the-crowd" (ATC) situation in the former case. It was observed that individual's net benefit is decreasing with respect to the entrance probability $r$, and the individual's best response is a decreasing function of the strategy selected by the other customers. In the fully observable case, we obtained that the individual's net benefit decreases with respect to the number of customers in the orbit, but the social welfare and the administrator's net benefit are concave with respect to the number of customers in the orbit.

Both in the partially observable case and the fully observable case, the solutions of the social maximization problem are smaller than the solutions of the individual equilibrium problem. This is aroused by the fact that the former customers imposed negative externality on the late arriving customers. Rational individuals in an economic system prefer to maximize their own benefit, but if customers want to maximize their social net benefit, they need to cooperate rather than ignore the negative externality that is imposed on the other customers. We observed that $r_{e} \geq$ $r_{\text {soc }} \geq r_{\text {prof }}$ in the partially observable case and $n_{\text {prof }} \leq$ $n_{\text {soc }} \leq n_{e}$ in the fully observable case, which mean that, when considering optimization problems, the effective service rate can not satisfy the customers' desirable service demand. This further implicates that there are some customers who can not obtain service in some cases even if they are identical.

The effect of the server unreliability on the system performance cannot be ignored. It was readily seen that customers' equilibrium entrance probability, optimal entrance probability, and the related threshold strategies all decrease as $\xi / \eta$ increases. This is very important in management process. When $\xi / \eta$ increases, some customers' interest will be undermined and decreases the social welfare and the administrator's net profit at the same time. So it is very crucial to improve the reliability of the sever to achieve the goal of optimal management. 


\section{Conflict of Interests}

The authors declare that there is no conflict of interests regarding the publication of this paper.

\section{Acknowledgments}

The research was supported by the National Natural Science Foundation of China (Grants nos. 11171019 and 713990334), and the Program for New Century Excellent Talents in University (no. NCET-11-0568).

\section{References}

[1] G. I. Falin and J. G. C. Templeton, Retrial Queues, Chapman \& Hall, London, UK, 1997.

[2] J. R. Artalejo and A. Gómez-Corral, Retrial Queueing Systems: A Computational Approach, Springer, Heidelberg, Germany, 2008.

[3] G. Fayolle, "A simple telephone exchange with delayed feedbacks," in Proceedings of the International Seminar on Teletraffic Analysis and Computer Performance Evalution, pp. 245-253, Amsterdam, The Netherlands, 1986.

[4] K. Farahmand, "Single line queue with repeated demands," Queueing Systems, vol. 6, no. 1, pp. 223-228, 1990.

[5] J. R. Artalejo and A. Gómez-Corral, "Steady state solution of a single-server queue with linear repeated requests," Journal of Applied Probability, vol. 34, no. 1, pp. 223-233, 1997.

[6] A. Economou and S. Kanta, "Equilibrium customer strategies and social-profit maximization in the single-server constant retrial queue," Naval Research Logistics, vol. 58, no. 2, pp. 107122, 2011.

[7] V. G. Kulkarni, "A game theoretic model for two types of customers competing for service," Operations Research Letters, vol. 2, no. 3, pp. 119-122, 1983.

[8] A. Elcan, "Optimal customer return rate for an $\mathrm{M} / \mathrm{M} / 1$ queueing system with retrials," Probability in the Engineering and Informational Sciences, vol. 8, no. 4, pp. 521-539, 1994.

[9] R. Hassin and M. Haviv, "On optimal and equilibrium retrial rates in a queueing system," Probability in the Engineering and Informational Sciences, vol. 10, no. 2, pp. 223-227, 1996.

[10] F. Zhang, J. Wang, and B. Liu, "On the optimal and equilibrium retrial rates in an unreliable retrial queue with vacations," Journal of Industrial and Management Optimization, vol. 8, no. 4, pp. 861-875, 2012.

[11] J. Wang and F. Zhang, "Strategic joining in $\mathrm{M} / \mathrm{M} / 1$ retrial queues," European Journal of Operational Research, vol. 230, no. 1, pp. 76-87, 2013.

[12] H. Li and Y. Q. Zhao, "A retrial queue with a constant retrial rate, server break downs and impatient customers," Stochastic Models, vol. 21, no. 2-3, pp. 531-550, 2005.

[13] A. Economou and S. Kanta, "Equilibrium balking strategies in the observable single-server queue with breakdowns and repairs," Operations Research Letters, vol. 36, no. 6, pp. 696-699, 2008.

[14] J. H. Cao and K. Cheng, "Analysis of an $M / G / 1$ queueing system with repairable service station," Acta Mathematicae Applicatae Sinica, vol. 5, no. 2, pp. 113-127, 1982. 


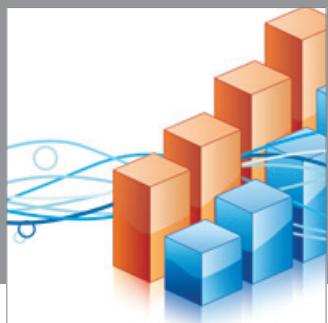

Advances in

Operations Research

mansans

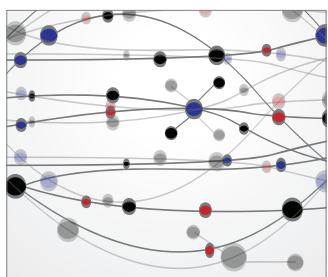

The Scientific World Journal
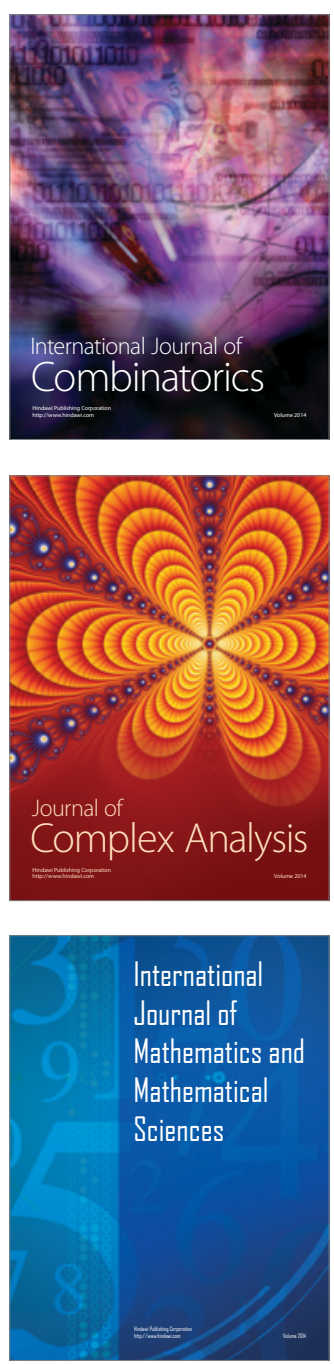
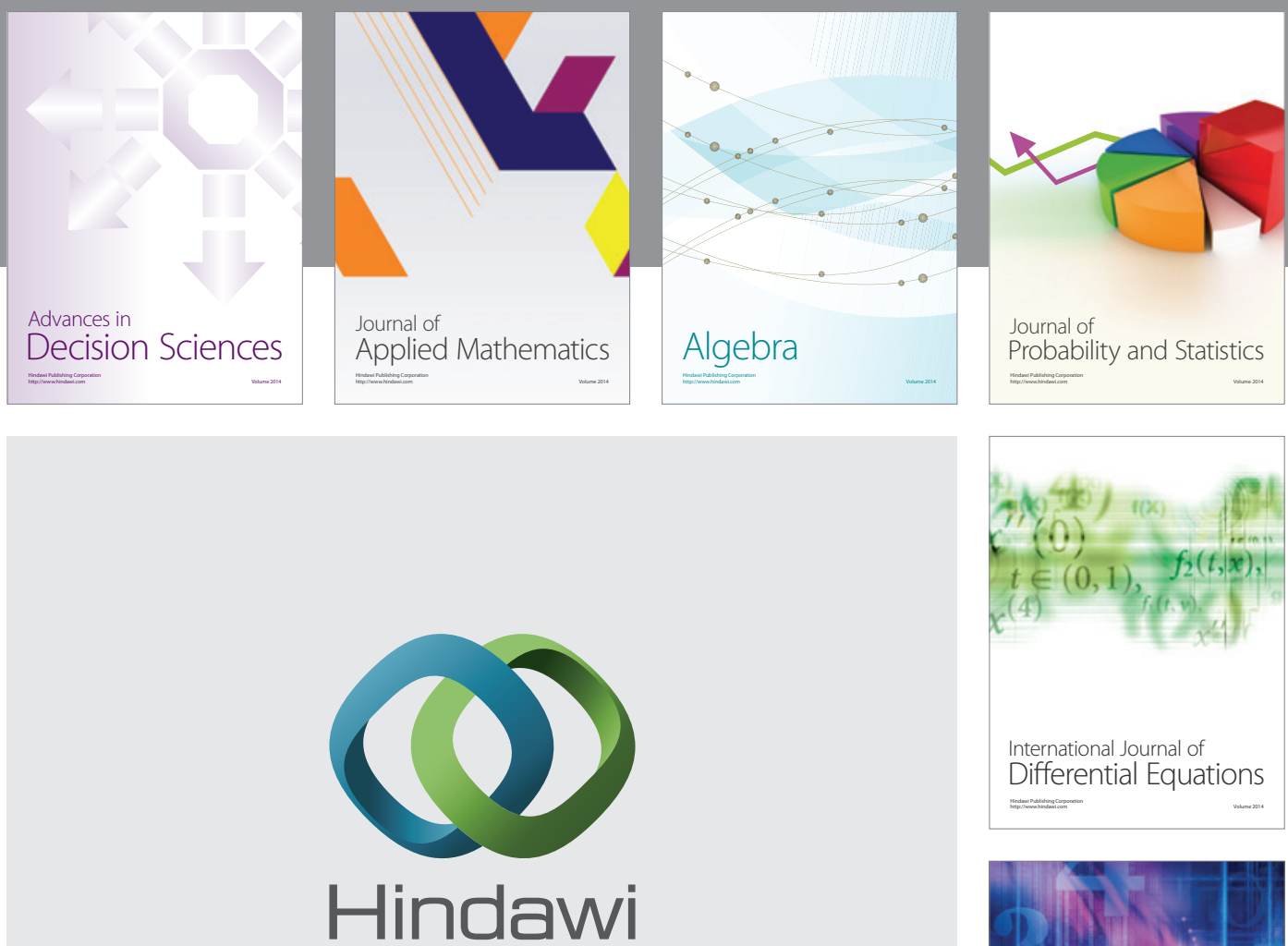

Submit your manuscripts at http://www.hindawi.com
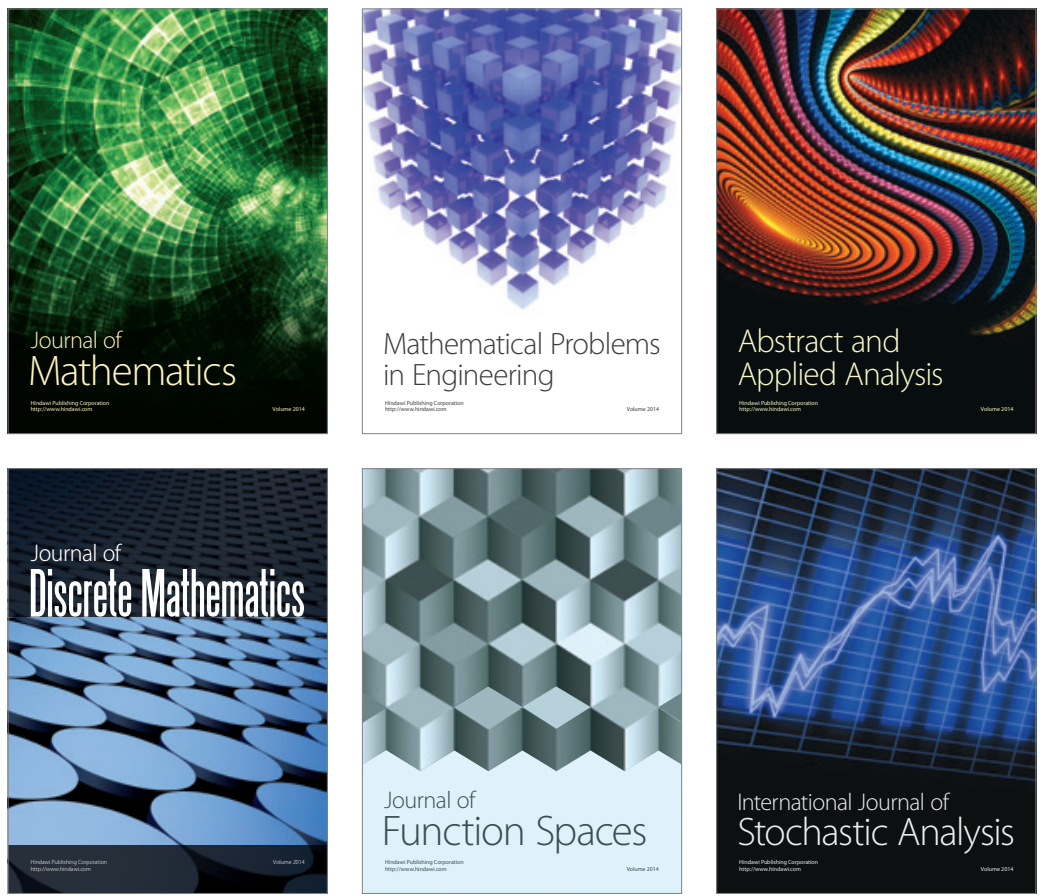

Journal of

Function Spaces

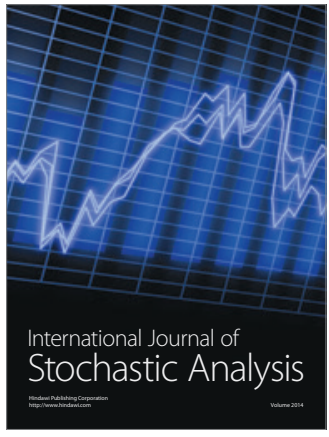

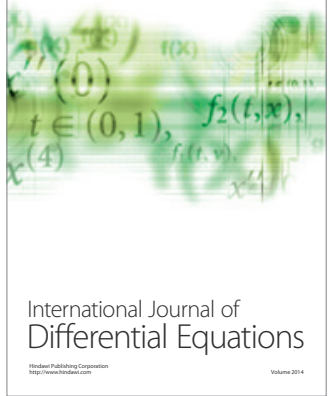
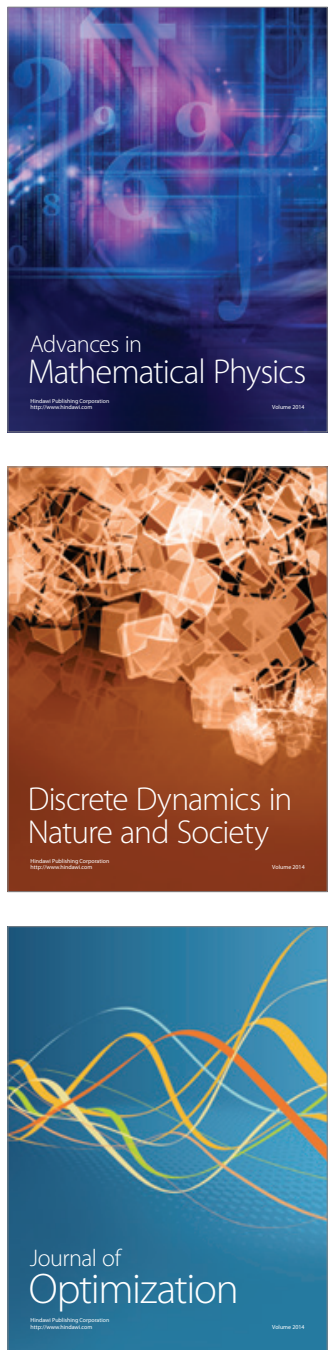